\title{
Photorefractive keratectomy after DMEK for corneal decompensation by phakic IOL
}

European Journal of Ophthalmology $1-4$

(C) The Author(s) 2022 Article reuse guidelines: sagepub.com/journals-permissions DOI: 10.1 I 77/II20672I211073429 journals.sagepub.com/home/ejo

(S)SAGE

\author{
Nuno Moura-Coelho',2,3 (D), Felicidad Manero', Renato Papa', \\ Nicolas Amich', João Paulo Cunha ${ }^{2,4}$ and José Luis Güell ${ }^{1,5}$
}

\begin{abstract}
Purpose: To provide the first description of photorefractive keratectomy (PRK) for the correction of mild residual refractive error after Descemet membrane endothelial keratoplasty (DMEK).

Methods: Case report.

Results: A 45 year-old woman presenting with phakic intraocular lens (PIOL)-related corneal decompensation underwent staged DMEK surgery following PIOL explantation and cataract surgery. Eighteen months after DMEK, uncorrected distance visual acuity (UDVA) was 20/60 and best-corrected visual acuity (BCVA) was 20/22, with a stable refraction. The patient requested refractive surgery to decrease spectacle dependance, and wavefront-optimized PRK was performed. At last follow-up observation thirty-three months after PRK (54 months after DMEK surgery), UDVA was 20/20, the cornea remained clear without signs of rejection or endothelial failure, and the endothelial cell loss rate was not accelerated after PRK.

Conclusion: Since long-term visual and refractive stability can be expected after DMEK, PRK may be a particular safe and effective approach for the correction of mild residual refractive errors after DMEK. However, we consider that surgeons must exercise caution when considering keratorefractive surgery in these eyes due to postoperative changes in corneal curvature and thickness, and further studies are encouraged.
\end{abstract}

\section{Keywords}

Photorefractive keratectomy, keratorefractive surgery, Descemet membrane endothelial keratoplasty

Date received: 4 August 2021; accepted: 6 December 202I

\section{Introduction}

Phakic intraocular lens (PIOL)-related corneal decompensation is an increasingly frequent indication for Descemet's membrane endothelial keratoplasty (DMEK). These are patients who have high visual demands and high expectation of spectacle independence; this is also true in patients who undergo DMEK after refractive crystalline lens surgery. Although DMEK has been considered a refractive neutral procedure, endothelial keratoplasty is associated with mild hyperopic shift, with an overall mean change in spherical equivalent of $+0.31 \mathrm{D}$ and a mean change in astigmatism ranging from -0.60 to $+1.11 \mathrm{D}$ after DMEK. ${ }^{1}$ Of note, DMEK may induce a refractive change $\geq 1.00 \mathrm{D}$ in up to $35 \%$ of patients, ${ }^{2}$ and up to $32 \%$ of patients experience a change in astigmatism higher than $1.00 \mathrm{D}$ after DMEK, related to the
'Cornea and Refractive Surgery Department, Instituto de Microcirugía Ocular (IMO) Barcelona, Barcelona, Spain

${ }^{2}$ Cataract and Refractive Surgery Unit, Hospital CUF Cascais, Cascais, Portugal

${ }^{3}$ NOVA Medical School, Faculdade de Ciências Médicas, Universidade Nova de Lisboa (NMS|FCM-UNL), Lisbon, Portugal

${ }^{4}$ Escola Superior de Tecnologías da Saúde de Lisboa (ESTeSL), Lisbon, Portugal

${ }^{5}$ Universidad Autónoma de Barcelona (UAB), Barcelona, Spain

\section{Corresponding author:}

Nuno Moura-Coelho, Cornea and Refractive Surgery Department Instituto de Microcirugía Ocular (IMO) Barcelona, Carrer Josep María Lladó, 3, Barcelona, Cataluña 08035, Spain; Cataract and Refractive Surgery Unit, Hospital CUF Cascais, R. Fernão Lopes 60, 2750-663 Cobre, Cascais, Portugal; NOVA Medical School, Faculdade de Ciências Médicas, Universidade Nova de Lisboa (NMS|FCM-UNL), Campo dos Mártires da Pátria 130, $1169-056$ Lisbon, Portugal.

Email: nunomouracoelho.oft@gmail.com 
combination of postoperative corneal deswelling and corneal incision-induced astigmatism. ${ }^{3}$ However, longterm visual and refractive stability can be expected after DMEK, which has risen the question as to whether these cases are amenable to keratorefractive surgery, as has been reported in Descemet stripping endothelial keratoplasty. ${ }^{4,5}$ This suggests that photorefractive keratectomy (PRK) may be a safer approach to correct the residual refractive error after DMEK.

We present the first reported case of wavefront-optimized photorefractive keratectomy (WFO-PRK) after DMEK. Written informed consent was obtained from the patient and the study was performed according to the tenets of the Declaration of Helsinki.

\section{Case description}

A 45 year-old female patient underwent staged DMEK for corneal decompensation due to accelerated endothelial cell loss and cortical cataract four years after implantation of an angle-supported, anterior chamber PIOL in another center for correction of $-8.00 \mathrm{D}$ myopia; DMEK was performed 8 weeks after combined PIOL explantation plus cataract extraction and pseudophakic IOL implantation. Manifest refraction at the time of DMEK surgery was -0.50 cyl @ $20^{\circ}$, with a best-corrected visual acuity (BCVA) of $0.52 \log$ MAR (20/60 Snellen equivalent).

Postoperative cystoid macular edema was detected at 4-week follow-up after DMEK, which resolved after intravitreal sustained-release 700- $\mu \mathrm{g}$ dexamethasone implant. At 6-month follow-up after DMEK, BCVA was 0.10 $\log$ MAR (20/25 Snellen), with a residual refractive error of $-0.50 \mathrm{sph}-1.25 \mathrm{cyl} @ 100^{\circ}$, and at 18-month follow-up BCVA was $0.04 \log$ MAR (20/22 Snellen) with a stable manifest refraction, with uncorrected distance visual acuity (UDVA) of $0.52 \log$ MAR (20/60 Snellen). The patient requested surgery to improve spectacle independence. Corneal topography showed a thinnest point of $472 \mu \mathrm{m}$, with keratometry $44.30 \mathrm{D}$ and $45.80 \mathrm{D} @ 45^{\circ}$ (Figure 1). WFO-PRK was performed using the MEL 90 excimer LASER (Carl Zeiss Meditec, Jena, Germany), with a $6.0-\mathrm{mm}$ optical zone diameter.

At the completion of the procedure, a therapeutic soft contact lens was placed for 5 days, during which topical ofloxacin was instilled $\mathrm{q} 4 \mathrm{~h}$ in addition to a fixed combination of tobramycin $0.3 \%+$ dexamethasone $0.1 \% \mathrm{q} 4 \mathrm{~h}$. After contact lens removal, postoperative treatment was, as our standard, consisting of fixed combination of tobramycin $0.3 \%+$ dexamethasone $0.1 \%$ $\mathrm{q} 4 \mathrm{~h}$ during the first week and tapered over 3 weeks, then switched to fluorometholone $0.1 \% \mathrm{q} 8 \mathrm{~h}$ for 2 weeks with tapering over three months and then kept once daily indefinitely for post-DMEK rejection prophylaxis; a fixed combination of dexamethasone $0.05 \%+$ chloramphenicol $1 \%$ ointment q.h.s. for 3 months; and timolol $0.5 \%$ eyedrops $\mathrm{q} 12 \mathrm{~h}$ for 3 months.

No postoperative complications were observed after PRK. Three weeks after PRK, UDVA was $\operatorname{logMAR} 0$ (20/20 Snellen) with plano refraction, ant thirty-three months after PRK (54 months post-DMEK), UDVA was 0 logMAR (20/20 Snellen) and refraction remained plano. The cornea remained clear, without signs of allograft rejection or endothelial failure. Endothelial cell density (ECD) was 1704 cells $/ \mathrm{mm}^{2}$ (preoperative donor ECD decrease $=43 \%$ ) one year after PRK, and 1045 cells $/ \mathrm{mm}^{2}$ (preoperative donor ECD decrease $=$ $65 \%$ ) at 54-month after DMEK (Figure 2), which is in agreement with the linear decrease in ECD in the medium to long-term after DMEK. ${ }^{6}$

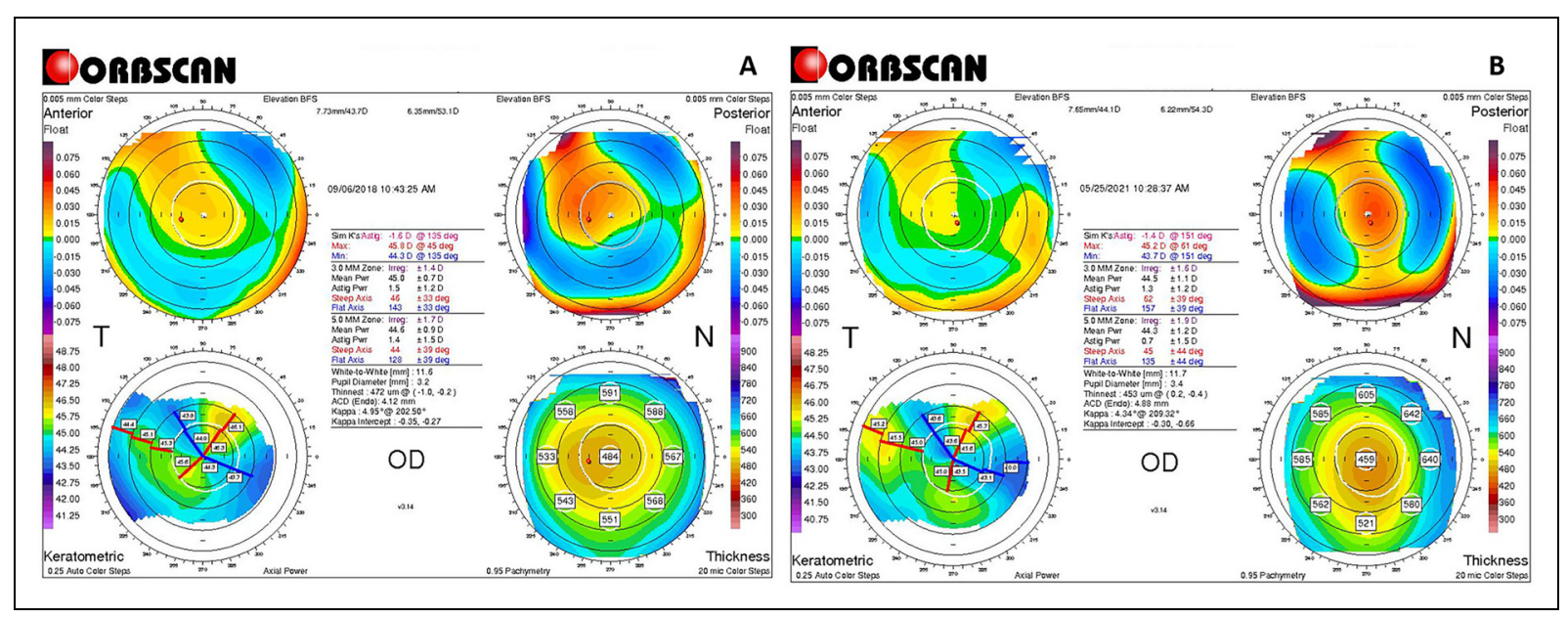

Figure I. (A) Corneal topography before wavefront-optimized photorefractive keratectomy (PRK). (B) Corneal topography 33 months after PRK (54 months after DMEK), showing keratometric stability. 


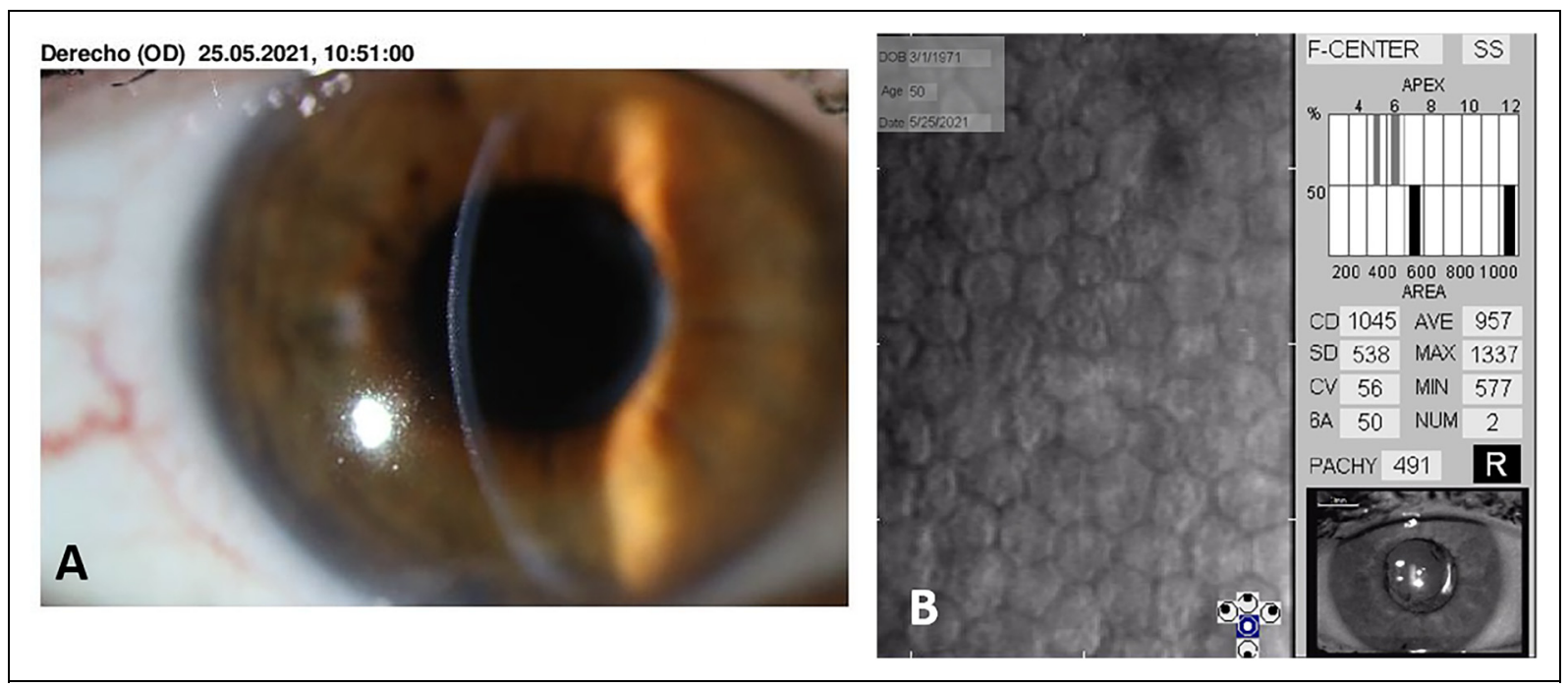

Figure 2. (A) Slit-lamp photograph showing corneal transparency maintained at last follow-up (54 months after DMEK). (B) Specular microscopy at last follow-up 33 months after photorefractive keratectomy, showing stable postoperative endothelial cell density.

\section{Conclusions}

Since the introduction of endothelial keratoplasty techniques, there has been an increasing interest in its refractive outcomes. $^{7}$ This is especially true in cases where DMEK is combined with refractive correction or cataract surgery, where the refractive target may be adjusted to account for the anticipated refractive shifts following DMEK. However, the literature is very limited regarding the treatment of post-DMEK refractive errors by means of laser refractive surgery. Price et al. reported the first case of laser-assisted in situ keratomileusis (LASIK) after DMEK, and raised doubt regarding the safety of excimer laser surgery in these eyes. ${ }^{8}$ Since then, only one small case series reported the outcomes of femtosecond-assisted LASIK after DMEK in eyes with Fuchs' corneal endothelial dystrophy. ${ }^{9}$ In that study, LASIK was found to be safe and effective. ${ }^{9}$

In our case, we have observed good midterm visual, refractive, and keratometric stability following PRK. To the best of our knowledge, this is the first published report of PRK for the correction of post-DMEK refractive error. Importantly, the DMEK graft ECD loss was not accelerated after PRK, in line with previous studies that donor graft ECD after DSAEK or DMEK is unlikely to be affected by excimer laser treatment. ${ }^{4,9}$

In eyes with refractive changes $>1.00 \mathrm{D}$ following DMEK, the magnitude of refractive "surprise" reported in previous publications has ranged from 1.25 to $2.50 \mathrm{D}$ in astigmatism, and nearly $90 \%$ of patients will be within $2.00 \mathrm{D}$ of emmetropia. We believe that this amount of residual refractive error may be amenable to PRK in many cases. However, PRK may not be effective in all cases, and we still consider that surgeons must exercise caution when considering keratorefractive surgery in DMEK eyes. After DMEK, changes in both the anterior and posterior corneal curvatures can occur. ${ }^{1}$ Resolution of corneal edema after DMEK may be associated with a thinner-than-normal central cornea, steeper pachymetric progression from the thinnest point to the periphery, and higher posterior elevation values. ${ }^{10}$ Further long-term prospective studies are encouraged to adequately assess the stability of this surgery as well as the safety of the procedure.

\section{Declaration of conflicting interests}

The author(s) declared no potential conflicts of interest with respect to the research, authorship, and/or publication of this article.

\section{Funding}

The author(s) received no financial support for the research, authorship and/or publication of this article.

\section{Authorship}

Nuno Moura-Coelho: conceptualization, methodology, data collection, writing original draft, writing review and editing. Felicidad Manero: data collection, writing original draft, supervision, validation. Renato Papa: writing review and editing; Nicolas Amich: writing original draft; João P Cunha: writing review and editing, supervision, validation; José L Güell: conceptualization, methodology, project administration, writing review and editing, supervision, validation. All authors attest that the meet the current ICMJE criteria for authorship.

\section{ORCID iD}

Nuno Moura-Coelho (iD https://orcid.org/0000-0002-5176-1411 


\section{References}

1. Deng SX, Lee WB, Hammersmith KM, et al. Descemet membrane endothelial keratoplasty: safety and outcomes: a report by the American academy of ophthalmology. Ophthalmology 2018; 125: 295-310.

2. van Dijk K, Rodriguez-Calvo-de-Mora M, van Esch H, et al. Two-year refractive outcomes after descemet membrane endothelial keratoplasty. Cornea 2016; 35: 1548-1555.

3. Ham L, Dapena I, Moutsouris K, et al. Refractive change and stability after Descemet membrane endothelial keratoplasty. Effect of corneal dehydration-induced hyperopic shift on intraocular lens power calculation. J Cataract Refract Surg 2011; 37: 1455-1464.

4. Ratanasit A and Gorovoy MS. Laser-assisted in situ keratomileusis or photorefractive keratectomy after descemet stripping automated endothelial keratoplasty. Cornea 2011; 30: 787-789.

5. Fung SS, Iovieno A, Shanmuganathan V, et al. Femtosecond laser refractive surgery after descemet stripping-automated endothelial keratoplasty. Case Rep Ophthalmol Med. Epub ahead of print 2012 April 18. 2012; 2012: 190953.

6. Vasiliauskaite I, Oellerich S, Ham L, et al. Descemet membrane endothelial keratoplasty: ten-year graft survival and clinical outcomes. Am J Ophthalmol 2020; 217: 114-120.

7. Tong CM, Baydoun L and Melles GRJ. Descemet membrane endothelial keratoplasty and refractive surgery. Curr Opin Ophthalmol 2017; 28: 316-325.

8. Price FW, Price MO and Guerra F. Is excimer laser corneal surgery appropriate after resolution of corneal edema in Fuchs dystrophy by Descemet membrane endothelial keratoplasty? J Refract Surg 2011; 27: 299-302.

9. Fernández-Vega-Cueto L, Lisa $\mathrm{C}$, Naveiras $\mathrm{M}$, et al. FemtoLASIK after Descemet membrane endothelial keratoplasty. Cornea 2020; 39: 468-472.

10. Arnalich-Montiel F, Mingo-Botín D and Diaz-Montealegre A. Keratometric, pachymetric, and surface elevation characterization of corneas with Fuchs endothelial corneal dystrophy treated with DMEK. Cornea 2019; 38: 535-541. 